Revista de Investigación Universitaria | Volumen 6 - Número 2, Julio - Diciembre, 2017 | ISSN 2078-4015 (En línea)

DOI:https://doi.org/10.17162/riu.v6i2.1074

(cc) BY

\title{
CALIDAD DEL SERVICIO DE LAS RESIDENCIAS UNIVERSITARIAS Y LA SATISFACCIÓN DE LOS ESTUDIANTES INTERNOS EN UNA UNIVERSIDAD PRIVADA CONFESIONAL
}

\author{
Mirla Carrera Fernández*a \\ aUniversidad Peruana Unión, Lima - Perú \\ Recibido: 10 de marzo de 2017 Aceptado: 20 de abril de 2017
}

\begin{abstract}
Resumen
El objetivo de esta investigación es determinar la relación que existe entre la calidad del servicio de las residencias universitarias y la satisfacción de los estudiantes internos de una universidad privada confesional. La investigación es descriptiva, correlacional, no experimental de diseño transversal. Para medir la calidad del servicio se utilizó el modelo servqual, y para medir la satisfacción se utilizó un instrumento basado en los elementos de satisfacción de Kotler. La muestra es de 225 estudiantes internos pertenecientes a las residencias de la universidad privada confesional. Para determinar la correlación se utilizó el coeficiente de correlación Rho de Spearman. Los resultados mostraron un p-valor (Sig.) de 0,000 y un coeficiente de correlación de 0.778. Por lo tanto, se llegó concluyó que existe relación significativa y fuerte entre la calidad del servicio de las residencias universitarias y la satisfacción de los estudiantes internos de la universidad privada confesional
\end{abstract}

Palabras claves: Calidad de servicio, residencias universitarias, satisfacción, relación, estudiantes

\begin{abstract}
The aim of this research is to find the relationship between the quality of the service of the university residences and the satisfaction of the internal students at the Universidad Peruana Unión in Lima, 2017. This is a descriptive, correlational research with a noexperimental and transversal design. Servqual's model was used to measure the quality of the service, and an instrument based on Koller's satisfaction elements was used to measure satisfaction. The sample size was 225 internal students belonging to the residences of the Universidad Peruana Unión in Lima. To find the correlation, Spearman's Rho correlation coefficient was used. The results showed a p-value (Sig.) of 0.000 and a correlation coefficient of 0.778. Therefore, it was concluded that there is a significant and strong relationship between the quality of the service of the university residences and the satisfaction of the internal students at the Universidad Peruana Unión in Lima.
\end{abstract}

Keywords: Quality of service, university residences, satisfaction, relationship, students

${ }^{\star}$ Email: mirlacf@hotmail.com 
Revista de Investigación Universitaria | Volumen 6 - Número 2, Julio - Diciembre, 2017 | ISSN 2078-4015 (En línea)

DOI :

(cc) Br

\section{Introducción}

La competitividad entre empresas y el aumento de las exigencias de los consumidores ha sido el motor de arranque para que las organizaciones busquen un factor diferenciador para mantenerse en el mercado, es por ello que la calidad en la actualidad se ha convertido en una necesidad para las estrategias que tienen como finalidad el éxito y supervivencia de las empresas. La calidad no es responsabilidad solamente de un area de la organización, Atencio \& González (2007) afirman que la calidad es responsabilidad de toda la organización pues todos directa - indirectamente están involucrados con el cliente y el factor humano en las empresas es clave para que el cliente perciba la calidad, además de otros factores que también influyen como la tecnología, las relaciones comerciales y el medio ambiente (Alcalde, 2007). Parasuraman, Zeithaml, \& Berry (1988) definieron la calidad del servicio como un juicio global del consumidor, la cual resulta de la comparación que el cliente realiza de sus expectativas sobre el servicio que recibirán y la percepción de la actuación de las empresas proveedoras de ese servicio. Keller, (2006) define la satisfacción como la comparación que realiza un cliente a partir del resultado que obtiene de un producto con respecto a la expectativa que tenía del mismo. Ambas tienen que ver con el bienestar del cliente. Morillo \& Morillo (2016) manifiestan que la calidad del servicio y la satisfacción aseguran relaciones de fidelidad entre el proveedor del servicio y el usuario. Un cliente satisfecho traerá como resultados beneficios para la empresa Hoffman \& Bateson (2002) mencionan que estos beneficios son: publicidad boca a boca, constancia, no habrá presión ante la competencia y un buen ambiente laboral. 
Revista de Investigación Universitaria | Volumen 6 - Número 2, Julio - Diciembre, 2017 | ISSN 2078-4015 (En línea)

DOI :

La Universidad Peruana Unión es una entidad de estudios superiores, la cual cuenta con el servicio de residencias universitarias; 2 residencias para varones y 3 para señoritas en estas residencias se atienden un aproximado de 460 estudianes internos, durante los periodos académicos. Los cupos libres en los internados es de aproximadamente un 27\% anual; ya sea por retiros, porque no ingresaron suficientes estudiantes para cubrir la capacidad máxima, entre otros posibles casos. De esta manera es interés de esta investigación, tomar conocimiento de las características que promueven la demanda de los servicios de las residencias en la UPeU, así como de forma particular la satisfacción de los estudiantes internos en relación a los servicios ofrecidos, orientados por los criterios de una mejora continua.

Por lo antes expuesto, consideramos apropiado desarrollar la presente investigación, con la pretensión de determinar la relación que existe entre la calidad del servicio que ofrecen las residencia de la Universidad Peruana Unión sede Lima y la satisfacción de los estudiantes internos, beneficiarios del servicio.

\section{Diseño metodológico}

Esta investigación es de tipo descriptivo y correlacional. Descriptivo porque mide de forma independiente las variables (Arias, 1999). Correlacional pues examina relaciones entre las variables de estudio (Bernal Torres, 2006). Es no experimental, pues no se manipulan las variables sino que se analizan según su contexto natural; se describen, determinan y explican tal cual en el campo de estudio (Toro Jaramillo \& Parra Ramírez, 2006). El diseño de la investigación es transversal porque se obtuvo información del objeto de 
Revista de Investigación Universitaria | Volumen 6 - Número 2, Julio - Diciembre, 2017 | ISSN 2078-4015 (En línea)

DOI :

estudio una única vez, que en este caso fue una muestra de la población de estudiantes internos de las residencias de la Universidad Peruana Unión sede Lima, 2017 (Bernal Torres, 2006). La población esta conformada por 543 estudiantes internos de la Universidad Peruana Unión sede Lima, 2017; de los cuales 289 son varones y 254 señoritas. La muestra de la población esta conformada por 225 estudiantes internos de la Universidad Peruana Unión sede Lima, 2017. Para el cumplimiento de los objetivos de esta investigación, se aplicó la encuesta como técnica de recolección de la información, el cual ha ayudado a identificar las relaciones entre la calidad del servicio y la satisfacción. Para la variable calidad del servicio se ha utilizado el instrumento de evaluación SERVQUAL, el cual cuenta con 22 ítems distribuidos en 5 dimensiones de la manera siguiente: 4 ítems en elemento tangibles, 5 ítems en fiabilidad, 4 ítems en capacidad de respuesta, 4 ítems en seguridad y 5 ítems en empatía. El instrumento utilizado para medir la variable calidad del servicio tuvo un alfa de Cronbach de 0.948 evidenciando su fiabilidad. Para la variable satisfacción se aplicó un instrumento de evaluación según los elementos de la satisfacción según Kotler, este instrumento tuvo un alfa de Cronbach de 0.933 evidenciando su fiabilidad.

\section{Resultados}

\section{Relación entre calidad del servicio y satisfacción}

La figura 1, evidencia una distribución no normal de la variable calidad de servicio, debido a que muestra asimetria hacia la izquierda y no hay una concentración de los datos dentro de la campana de Gauss, la desviación estándar es de 14.44 y le media de 77.91 . 
Revista de Investigación Universitaria | Volumen 6 - Número 2, Julio - Diciembre, DOI :

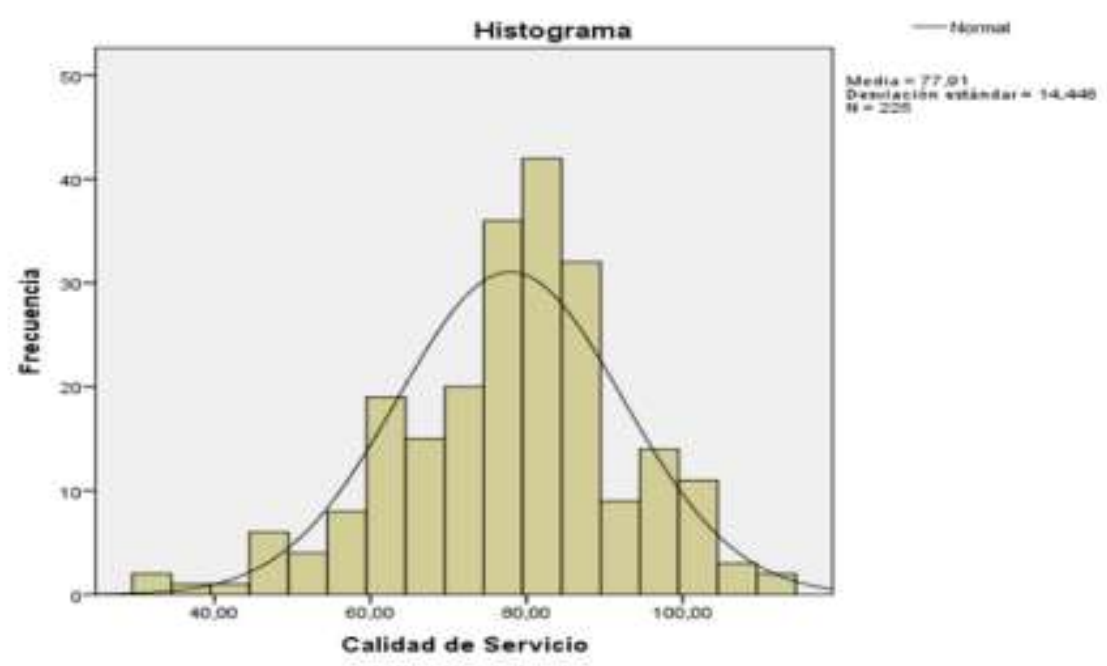

servicio

Figura 1: Prueba de normalidad calidad de

La figura 2, muestra una distribución no normal de la variable satisfacción, debido a que muestra asimetria hacia la izquierda, lo cual indica que la concentración de la muestra encuestada no se encuentra en la media y no se ajusta a la línea de distribución, la desviación estándar es de 11.16 y le media de 60.38 .

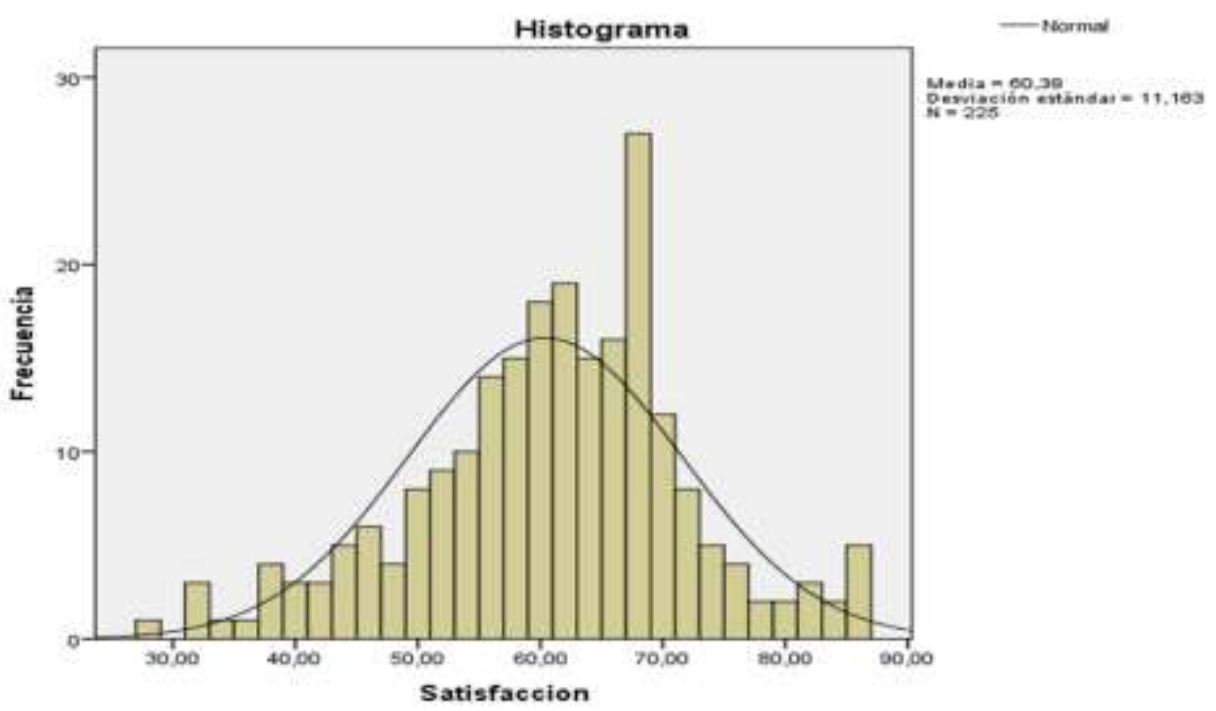

Figura 2. Prueba de normalidad satisfacción

\section{Prueba de hipótesis}

$\mathrm{H}_{0}$ : La calidad de servicio y satisfacción tienen una distribución normal. 
Revista de Investigación Universitaria | Volumen 6 - Número 2, Julio - Diciembre, DOI :

H: La calidad de servicio y satisfacción no tienen una distribución normal.

\section{Decisión:}

Si el p-valor (sig.) > 0.05 se acepta la hipótesis nula $\left(\mathrm{H}_{0}\right)$ •

Si el p-valor (sig.) < 0.05 se rechaza la hipótesis nula ( $\left.\mathrm{H}_{0}\right)$ y se acepta la alterna $\left(\mathrm{H}_{1}\right)$.

La tabla 1, presenta los resultados de la prueba de normalidad para la calidad de servicio y satisfacción aplicando el estadístico del contraste Kolmogorov Smirnov, para una muestra > 50, con un p-valor (sig.) de $0.001<0.05$ para la calidad de servicio, p-valor (sig.) de $0.005<0.05$ para satisfacción; por lo que se rechaza la hipótesis nula y se acepta la alterna, evidenciando que la calidad de servicio y satisfacción derivan de una distribución no normal. Por lo cual se aplico el coeficiente de correlación de spearman para hallar la relación entre ambas variables.

\begin{tabular}{|c|c|c|c|}
\hline & \multicolumn{3}{|c|}{ Kolmogorov-Smirnova } \\
\hline & Estadístico & $g 1$ & Sig. \\
\hline $\begin{array}{c}\text { Calidad de } \\
\text { Servicio }\end{array}$ & .083 & 225 & .001 \\
\hline Satisfacción & .073 & 225 & .005 \\
\hline
\end{tabular}

\section{Prueba de hipótesis}

$\mathrm{H}_{0}$ : No existe relación entre la calidad del servicio de las residencias universitarias y la satisfacción de los estudiantes internos de la Universidad Peruana Unión sede Lima, 2017.

$\mathbf{H}_{1}$ : Existe relación entre la calidad del servicio de las residencias universitarias y la satisfacción de los 
Revista de Investigación Universitaria | Volumen 6 - Número 2, Julio - Diciembre, DOI :

estudiantes internos de la Universidad Peruana Unión sede Lima, 2017.

\section{Decisión}

Si el p-valor (sig.) > 0.05, se acepta la hipótesis nula $\left(\mathrm{H}_{0}\right)$.

Si el p-valor (sig.) $>0.05$ se rechaza la hipótesis nula y se acepta la hipótesis alterna $\left(H_{1}\right)$.

La tabla 2, muestra un p-valor (Sig.) de 0,000<0,05, por lo que se rechaza hipótesis nula y se acepta la hipótesis alterna. Así mismo, presenta un coeficiente de correlación igual a 0,778 el cual indica que existe relación significativa y fuerte entre la calidad del servicio de las residencias universitarias y la satisfacción de los estudiantes internos de la Universidad Peruana Unión sede Lima, 2017. Esto confirma que existe dependecia ente ambas variables tal como dice Tigani (2006) que a mayor calidad en el servicio mayor satisfacción o viceversa.

Tabla 2. Aplicación del Rho de Spearman para determinar la relación entre la calidad del servicio (V1) y la satisfacción (V2)

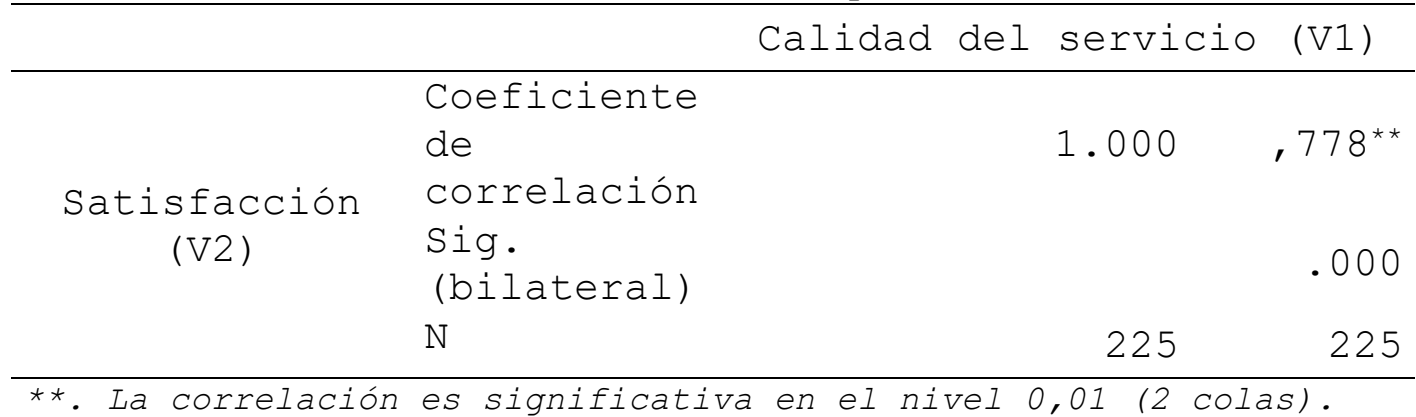

\section{Relación entre elementos tangibles y satisfacción Prueba de hipótesis}

$\mathrm{H}_{0}$ : No existe relación entre los elementos tangibles de las residencias universitarias y la satisfacción de los estudiantes internos de la Universidad Peruana Unión sede Lima, 2017.

$\mathbf{H}_{1}$ : Existe relación entre los elementos tangibles de las residencias universitarias y la satisfacción de los 
Revista de Investigación Universitaria | Volumen 6 - Número 2, Julio - Diciembre, 2017 | ISSN 2078-4015 (En línea)

DOI :

estudiantes internos de la Universidad Peruana Unión sede Lima, 2017.

\section{Decisión:}

Si el p-valor (sig.) > 0.05, se acepta la hipótesis nula $\left(\mathrm{H}_{0}\right)$.

Si el p-valor (sig.) < 0.05 se rechaza la hipótesis nula y se acepta la hipótesis alterna $\left(H_{1}\right)$.

La tabla 3, muestra un p-valor (Sig.) de 0,000<0,05, por lo que se rechaza hipótesis nula y se acepta la hipótesis alterna. Así mismo, presenta un coeficiente de correlación igual a 0,654 indicando que exite relación significativa y fuerte entre los elementos tangibles de las residencias universitarias y la satisfacción de los estudiantes internos de la Universidad Peruana Unión sede Lima, 2017. O sea, que los estudiantes internos perciben calidad en los elementos tangibles y por ello se sienten satisfechos.

Tabla 3. Aplicación del Rho de Spearman para determinar la relación entre los elementos tangibles (DIVI) y la satisfacción (V2)

Elementos tangibles

( D1V1)

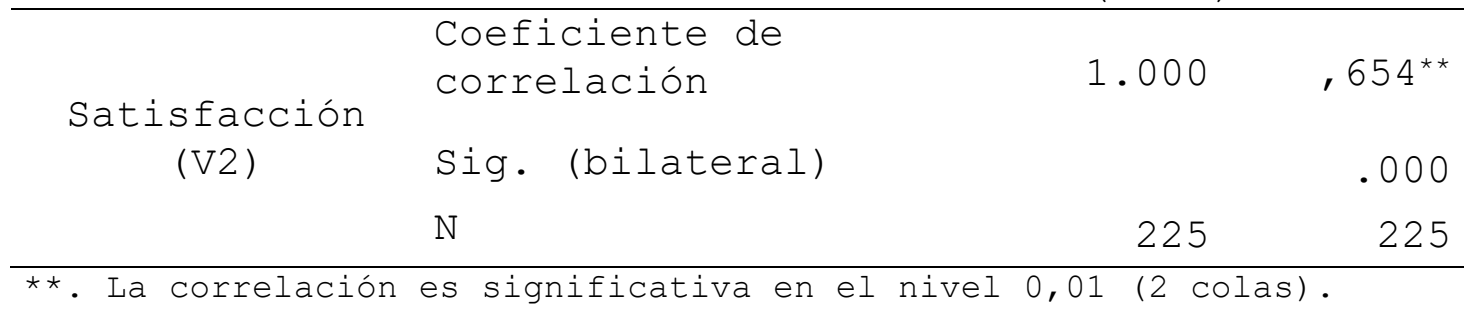

\section{Relación entre fiabilidad y satisfacción Prueba de hipótesis:}

$\mathbf{H}_{0}$ : No existe relación entre la fiabilidad de las residencias universitarias y la satisfacción de los estudiantes internos de la Universidad Peruana Unión sede Lima, 2017.

$\mathbf{H}_{1}$ : Existe relación entre la fiabilidad de las residencias universitarias y la satisfacción de los estudiantes internos de la Universidad Peruana Unión sede Lima, 2017. 
Revista de Investigación Universitaria | Volumen 6 - Número 2, Julio - Diciembre, DOI :

\section{(cc) $\mathrm{BY}$}

\section{Decisión:}

Si el p-valor (sig.) >0.05, se acepta la hipótesis nula $\left(\mathrm{H}_{0}\right)$.

Si el p-valor (sig.) < 0.05 se rechaza la hipótesis nula y se acepta la hipótesis alterna $\left(H_{1}\right)$.

La tabla 4, muestra un p-valor (Sig.) de 0,000<0,05, por lo que se rechaza hipótesis nula y se acepta la hipótesis alterna. Así mismo, presenta un coeficiente de correlación igual a 0,716 , lo cual indica que existe relación significativa y fuerte entre la fiabilidad de las residencias universitarias y la satisfacción de los estudiantes internos de la Universidad Peruana Unión sede Lima, 2017. Esto quiere decir que el aumento de la calidad en la fiabilidad significará un aumento de la satisfacción.

Tabla 4. Aplicación del Rho de Spearman para determinar la relación entre la fiabilidad (D2V1) y la satisfacción (V2)

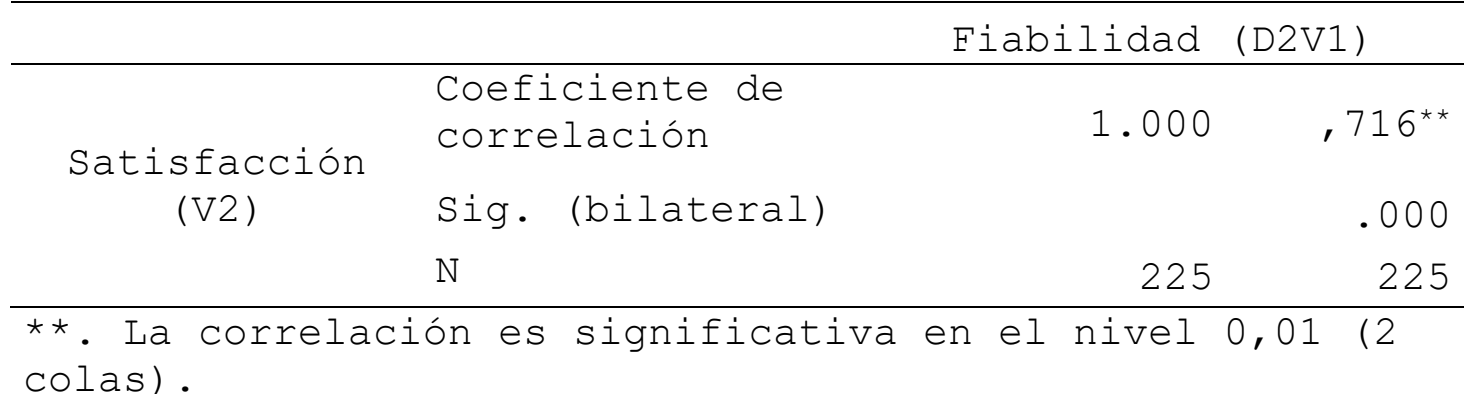

\section{Relación entre capacidad de respuesta y satisfacción Prueba de hipótesis}

$\mathrm{H}_{0}$ : No existe relación entre la capacidad de respuesta de las residencias universitarias y la satisfacción de los estudiantes internos de la Universidad Peruana Unión sede Lima, 2017.

$\mathbf{H}_{1}$ : Existe relación entre la capacidad de respuesta de las residencias universitarias y la satisfacción de los 
Revista de Investigación Universitaria | Volumen 6 - Número 2, Julio - Diciembre, 2017 | ISSN 2078-4015 (En línea)

DOI :

estudiantes internos de la Universidad Peruana Unión sede Lima, 2017.

\section{Decisión}

Si el p-valor (sig.) >0.05, se acepta la hipótesis nula $\left(\mathrm{H}_{0}\right)$.

Si el p-valor (sig.) > 0.05 se rechaza la hipótesis nula y se acepta la hipótesis alterna $\left(\mathrm{H}_{1}\right)$.

La tabla 5, muestra un p-valor (Sig.) de 0,000<0,05, por lo que se rechaza hipótesis nula y se acepta la hipótesis alterna. Así mismo, presenta un coeficiente de correlación igual a 0,616. Por lo tanto existe relación significativa y fuerte entre la capacidad de respuesta de las residencias universitarias y la satisfacción de los estudiantes internos de la Universidad Peruana Unión sede Lima, 2017. Esto quiere decir que a mayor percepción de calidad en la capacidad de respuesta mayor satisfacción.

\begin{tabular}{|c|c|c|c|}
\hline \multirow{4}{*}{$\begin{array}{c}\text { Satisfacción } \\
\text { (V2) }\end{array}$} & & \multicolumn{2}{|c|}{$\begin{array}{c}\text { Capacidad de respuesta } \\
\text { (D3V1) }\end{array}$} \\
\hline & $\begin{array}{l}\text { Coeficiente de } \\
\text { correlación }\end{array}$ & 1.000 &, $616^{\star \star}$ \\
\hline & Sig. (bilateral) & & .000 \\
\hline & $\mathrm{N}$ & 225 & 225 \\
\hline
\end{tabular}

\section{Relación entre seguridad y satisfacción Prueba de hipótesis:}

$\mathrm{H}_{0}$ : No existe relación entre la seguridad de las residencias universitarias y la satisfacción de los estudiantes internos de la Universidad Peruana Unión sede Lima, 2017. 
Revista de Investigación Universitaria | Volumen 6 - Número 2, Julio - Diciembre, 2017 | ISSN 2078-4015 (En línea)

DOI :

(cc) Br

$\mathbf{H}_{1}$ : Existe relación entre la seguridad de las residencias universitarias y la satisfacción de los estudiantes internos de la Universidad Peruana Unión sede Lima, 2017.

\section{Decisión:}

Si el p-valor (sig.) > 0.05, se acepta la hipótesis nula $\left(\mathrm{H}_{0}\right)$.

Si el p-valor (sig.) < 0.05 se rechaza la hipótesis nula y se acepta la hipótesis alterna $\left(\mathrm{H}_{1}\right)$.

La tabla 6, muestra un p-valor (Sig.) de 0,000<0,05, por lo que se rechaza hipótesis nula y se acepta la hipótesis alterna. Así mismo, presenta un coeficiente de correlación igual a 0,589, indicando que existe relación positiva y moderada entre la seguridad de las residencias universitarias y la satisfacción de los estudiantes internos de la Universidad Peruana Unión sede Lima, 2017. Esto quiere decir que un 41\% de los estudiantes internos que perciben calidad en la seguridad no necesariamente están satisfechos y/o viceversa.

Tabla 6. Aplicación del Rho de Spearman para determinar la relación entre la seguridad (D4V1) y la satisfacción (V2)

\begin{tabular}{|c|c|c|c|}
\hline & & Seguridad & (D4V1) \\
\hline \multirow{3}{*}{$\begin{array}{c}\text { Satisfacción } \\
\text { (V2) }\end{array}$} & $\begin{array}{l}\text { Coeficiente de } \\
\text { correlación }\end{array}$ & 1.000 &, $589^{\star *}$ \\
\hline & Sig. (bilateral) & & .000 \\
\hline & $\mathrm{N}$ & 225 & 225 \\
\hline
\end{tabular}

\section{Relación entre empatía y satisfacción Prueba de hipótesis:}

$\mathrm{H}_{0}$ : No existe relación entre la empatía de las residencias universitarias y la satisfacción de los estudiantes internos de la Universidad Peruana Unión sede Lima, 2017. 
Revista de Investigación Universitaria | Volumen 6 - Número 2, Julio - Diciembre, 2017 | ISSN 2078-4015 (En línea)

DOI :

(cc) Br

$\mathbf{H}_{1}$ : Existe relación entre la empatía de las residencias universitarias y la satisfacción de los estudiantes internos de la Universidad Peruana Unión sede Lima, 2017.

\section{Decisión:}

Si el p-valor (sig.) > 0.05, se acepta la hipótesis nula $\left(\mathrm{H}_{0}\right)$.

Si el p-valor (sig.) < 0.05 se rechaza la hipótesis nula y se acepta la hipótesis alterna $\left(\mathrm{H}_{1}\right)$.

La tabla 7, muestra un p-valor (Sig.) de 0,000<0,05, por lo que se rechaza hipótesis nula y se acepta la hipótesis alterna. Así mismo, presenta un coeficiente de correlación igual a 0,685 el cual indica que existe relación significativa y fuerte entre la empatía de las residencias universitarias y la satisfacción de los estudiantes internos de la Universidad Peruana Unión sede Lima, 2017. Esto significa que a mayor calidad en la empatía de las residencias universitarias mayor será la satisfacción de los estudiantes internos.

Tabla 7.Aplicación del Rho de Spearman para determinar la relación entre la empatía (D5V1) y la satisfacción (V2)

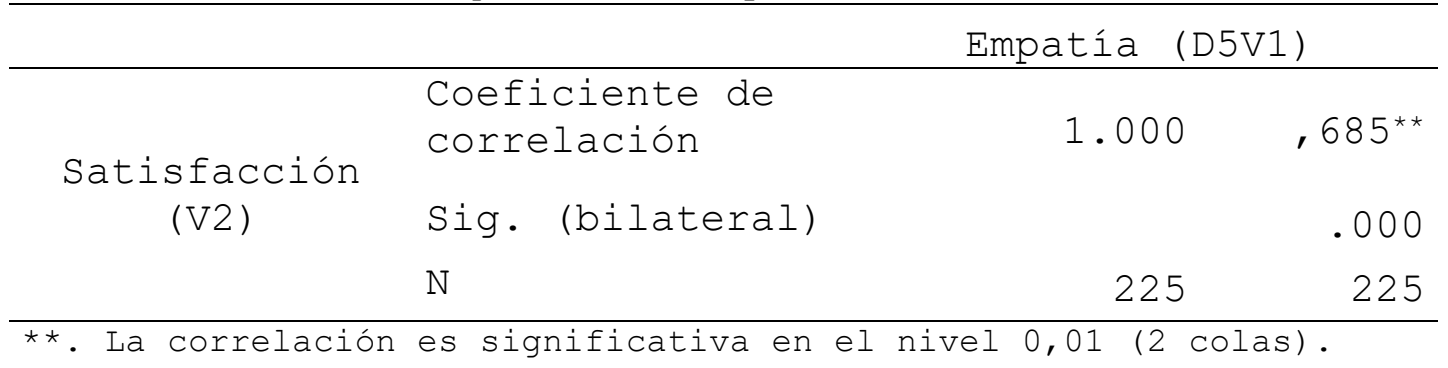

\section{Conclusiones}

En base al objetivo descrito en esta investigación, se determinó que existe relación significativa y fuerte entre la calidad del servicio de las residencias universitarias y la satisfacción de los estudiantes internos de la Universidad Peruana Unión sede Lima, 2017. Según el coeficiente de 
Revista de Investigación Universitaria | Volumen 6 - Número 2, Julio - Diciembre, 2017 | ISSN 2078-4015 (En línea)

DOI :

(cc) $\mathrm{Br}$

correlación un 78\% de los estudiantes internos consideran que existe calidad en los servicios en las residencias y por ende están satisfechos, esto quiere decir que la Universidad Peruana Unión sí cuenta con buenas residencias universitarias pero es necesario mantener el nivel de calidad y trabajar para continuar avanzando hasta conseguir que la percepción de la calidad sea mayor por parte de los estudiantes internos.

Con respecto a los elementos tangibles y la satisfacción se determinó que existe relación significativa y fuerte entre los elementos tangibles de las residencias universitarias y la satisfacción de los estudiantes internos de la Universidad Peruana Unión sede Lima, 2017. Se obtuvo un coeficiente de correlación de 0.685 y un sig. 0.000, o sea que los estudiantes internos perciben calidad en los elementos tangibles de las residencias y por ende están satisfechos, pero un 35\% de estudiantes internos no percibe lo mismo, esto indica que se necesita mejorar los muebles y/o equipos de las residencias, para hacerla mas atractiva de esta manera más estudiantes van a percibir calidad y por ende estarán satisfechos en lo que a tangibilidad se refiere; asi la relación entre ambas variables estará mas cerca a la perfección.

Según los resultados obtenidos, se determinó que existe relación significativa y fuerte entre la fiabilidad de las residencias universitarias y la satisfacción de los estudiantes internos de la Universidad Peruana Unión sede Lima, 2017. Para un 72\% de estudianes internos hay calidad en la fiabilidad de las residencias universitarias, esto muestra que las residencias están cumpliendo con su labor cubriendo las expectativas de un gran porcentaje de estudiantes internos no obstante a ello las residencias necesitan mejorar la rapidez en la solución de inconvenientes 
Revista de Investigación Universitaria | Volumen 6 - Número 2, Julio - Diciembre, 2017 | ISSN 2078-4015 (En línea)

DOI :

para que el 28\% de estudiantes internos restantes también perciban la calidad en la fiabilidad.

A través del analisis de correlación de spearman de la capacidad de respuesta y satisfacción, se determinó que existe relación significativa y fuerte entre la capacidad de respuesta de las residencias universitarias y la satisfacción de los estudiantes internos de la Universidad Peruana Unión sede Lima, 2017. Para un 62\% de estudiantes internos existe calidad en cuanto a capacidad de respuesta se refiere y por ello se sienten satisfechos, pero para un 38\% aun falta mejorar en este aspecto pues ellos sienten que el personal debe dedicarles tiempo cuando se les necesite.

Con respecto a la relación entre la seguridad y la satisfacción, se determinó que existe relación positiva moderada entre la seguridad de las residencias universitarias y la satisfacción de los estudiantes internos de la Universidad Peruana Unión sede Lima, 2017. Esto quiere decir que a pesar de que el estudiante interno se siente satisfecho con las residencias universitrias, se necesita fortalecer la confianza y credibilidad de los estudiantes internos hacia el desempeño del personal de las residencias, esto se lograra a través de capacitación al personal y conllevará a que el coeficiente de correlción se acerque más a la perfección.

En base a los resultados obtenidos en esta investigación se determinó que existe relación significativa y fuerte entre la empatía de las residencias universitarias y la satisfacción de los estudiantes internos de la Universidad Peruana Unión sede Lima, 2017. Con un coeficiente de correlación de 0.685 y un Sig. 0.00, lo cual significa que el estudiante interno percibe que se identifican con sus necesidades, pero a pesar de ello es necesario evaluar los 
Revista de Investigación Universitaria | Volumen 6 - Número 2, Julio - Diciembre, 2017 | ISSN 2078-4015 (En línea)

DOI :

horarios de actividades de las residencias y que estos horarios sean convenientes para los estudiantes internos.

\section{Recomendaciones}

Una vez descritos los resultados de la investigación se recomienda a la Gerencia de Servicios de la universidad privada confesional considerar lo siguiente:

1. Desarrollar un plan de mejora constante a través de la implementación de evaluaciones periódicas de la calidad del servicio y satisfacción.

2. Implementar un plan de capacitación para el personal del servicio en los internados que permita afianzar la confianza y credibilidad de los estudiantes internos, esto fortalecerá el sentido de seguridad en el estudiante interno.

\section{Referencias}

Alcalde, P. (2007). Calidad. (Paraninfo, Ed.). ThomsonParaninfo. Retrieved from

https://books.google.com.pe/books?id=M4KKceSe3f4C\&print sec $=$ frontcover \&dq=Calidad. Ediciones+paraninfo, + S.A. + Al calde, +pdf+gratis+2009\&hl=es$419 \& \mathrm{sa}=\mathrm{X} \& \mathrm{ved}=0$ ahUKEwj gjeCkmP7WAhUIiZAKHdp_D7UQ6AEIJDAA\# $\mathrm{V}=$ onepage $\& q \& f=f a l s e$

Arias, F. G. (1999). El Proyecto de Investigación, guía para su elaboración. (O. Ediciones, Ed.) (3ra edició). Caracas.

Atencio, E., \& González, B. (2007). Calidad de servicio en la editorial de la Universidad del Zulia (EDILUZ). Revista de Ciencias Sociales (RCS), XIII(1), 172-186.

Bernal Torres, C. A. (2006). Metodología de la investigación. (Pearson, Ed.) (Segunda ed). México.

Hoffman, K. D., \& Bateson, J. E. G. (2002). Marketing de servicios. (C. Learning, Ed.) (Cuarta Edi). México.

Keller, phlip K.-. (2006). Dirección de Marketingl - .

Morillo, M., \& Morillo, M. (2016). Satisfacción del usuario y calidad del servicio en alojamientos turísticos del estado Mérida, Venezuela. Revista de Ciencias Soiales (RCS), XXII(2), 111-131.

Parasuraman, A., Zeithaml, V., \& Berry, L. (1988). Servqual, a Multiple-item Scale for Measuring Consumer Perceptions of Service Quality. Journal of Retailing, 64, 12-40. 
Revista de Investigación Universitaria | Volumen 6 - Número 2, Julio - Diciembre, 2017 | ISSN 2078-4015 (En línea)

DOI :

(cc) BY

Tigani, D. (2006). Excelencia Excelencia en Servicio en Servicio.

Toro Jaramillo, I. D., \& Parra Ramírez, R. D. (2006). Método y conocimiento: metodología de la investigación: investigación cualitativa/investigación cuantitativa. Fondo Editorial Universitario EAFIT. Retrieved from https://books.google.com.pe/books?id=4Y-

kHGjEjy0C\&printsec=frontcover\&dq=Método+y+Conocimiento+ Metodologico+de+la+Investigación.\&hl=es-

$419 \& \mathrm{sa}=\mathrm{X} \& \mathrm{ved}=0$ ahUKEwj PtYqdtYXXAhWJDpAKHbPEB 4AQ 6AE I JDAA\# $\mathrm{v}=$ onepage $\& q=$ Método y Conocimiento Metodologico de la Investigación.\&f=false 